day functional outcome, mortality or stroke-onset to treatment compared with the directly presenting patients (all p>0.05). Successful reperfusion rates and $\mathrm{sICH}$ were similar between cohorts (all p>0.05)

Conclusion Inter-hospital transfer in the ETW but not LTW is associated with longer stroke-onset to treatment, worse 90-day functional outcome and higher mortality.

\section{ADJUNCTIVE INTRAARTERIAL THROMBOLYSIS IN ENDOVASCULAR CLOT RETRIEVAL: A SYSTEMATIC REVIEW AND META-ANALYSIS}

${ }^{1}$ William K Diprose, ${ }^{2}$ Michael TMTM Wang, ${ }^{1}$ Kaustubha Ghate, ${ }^{3}$ Stefan Brew, ${ }^{3}$ James R Caldwell, ${ }^{3}$ Ben McGuinness, ${ }^{2} \mathrm{P}$ Alan Barber. 'Department of Neurology, Auckland City Hospital, Auckland, New Zealand; 'Department of Medicine, University of Auckland, Auckland, New Zealand; ' ${ }^{3}$ Department of Radiology, Auckland City Hospital, Auckland, New Zealand

\subsection{6/bmjno-2021-ANZAN.28}

Objective To evaluate the safety and efficacy of intra-arterial thrombolysis (IAT) as an adjunct to endovascular clot retrieval (ECR) in ischaemic stroke, we performed a systematic review and meta-analysis of the literature.

Methods Searches were performed using Medline, Embase, and Cochrane databases for studies that compared ECR to ECR with adjunctive IAT (ECR+IAT). Safety outcomes included symptomatic intracerebral haemorrhage (sICH) and mortality at three months. Efficacy outcomes included successful reperfusion (Thrombolysis in Cerebral Infarction score of $2 \mathrm{~b}$ to 3 ), and functional independence, defined as a modified Rankin Scale score of 0 to 2 at three months.

Results Five studies were identified that compared combined ECR+IAT (IA alteplase or urokinase) to ECR-only, and were included in the random effects meta-analysis. There were 1693 ECR patients, including 269 patients treated with combined ECR+IAT and 1424 patients receiving ECR-only. Pooled analysis did not demonstrate any differences between ECR +IAT and ECR-only in rates of sICH (OR: 0.61, 95\% CI: $0.20-1.85 ; \mathrm{P}=0.78$ ), mortality (OR: $0.77,95 \% \mathrm{CI}: 0.54-1.10$; $\mathrm{P}=0.15$ ), or successful reperfusion (OR: 1.05 , 95\% CI: $0.52-$ $2.15 ; \mathrm{P}=0.89)$. There was a higher rate of functional independence in patients treated with ECR+IAT, although this was not statistically significant (OR: 1.34, 95\% CI: 1.00-1.80; $\mathrm{P}=0.053)$.

Conclusions Adjunctive IAT appears to be safe. In specific situations, neurointerventionists may be justified in administering small doses of intraarterial alteplase or urokinase as rescue therapy during ECR.

\section{HIGH SENSITIVITY TROPONIN IN ACUTE ISCHAEMIC STROKE STUDY (TACIS)}

${ }^{1}$ Andrew Hannaford, ${ }^{2}$ Michael Hayes, ${ }^{3}$ John Worthington, ${ }^{3}$ Timothy Ang, ${ }^{2}$ Nimalin Harinesan. 'Neurology, Westmead Hospital, Sydney, NSW, Australia; ${ }^{2}$ Neurology, Concord Repatriation General Hospital, Sydney, NSW, Australia; ${ }^{3}$ Neurology, Royal Prince Alfred Hospital, Sydney, NSW, Australia

\subsection{6/bmino-2021-ANZAN.29}

Objective We designed a multi-centre prospective cohort study to explore the hypothesis that early acutely elevated high sensitivity troponin (hsT) is associated with cardioembolic stroke (CES)
Methods Ischaemic strokes across three hospitals underwent hsTroponin testing and 2 blinded clinicians classified patients as CES, NCE (Non Cardioembolic) or ESUS by ESUS criteria. Characteristics included baseline NIHSS, renal function, hypertension, diabetes, smoking, ischaemic heart disease, past stroke and congestive cardiac failure. The odds of positive hsT for CES Vs NCE and ESUS Vs NCE were modelled with stepwise addition of patient characteristics.

Results 194 ischaemic stroke cases were included, with a mean age of 71 years and a 57:43 male:female ratio. 65 had a positive hsTroponin, which was associated with older age, hypertension, cardiac failure, coronary disease, an eGFR $<60$ and a higher NIHSS. Positive hsTroponin was associated with CES (OR, 2.06; 95\% CI, 1.12-3.79; $\mathrm{P}=0.02$ ). This association persisted after adjusting for confounders, such as age, sex, atrial fibrillation, renal impairment, ischaemic heart disease and previous stroke (Adjusted OR, 4.07; 95\% CI, 1.41-11.75; $\mathrm{P}=0.01)$. ESUS was negatively associated with an elevated hs troponin (OR, 0.45; 95\% CI, 0.22-0.94; $P=0.03$ ). This was not significant when adjusting for other variables $(\mathrm{P}=0.09)$.

Conclusions An elevated hs troponin after acute ischaemic stroke is independently associated with a cardioembolic mechanism. High sensitivity cardiac troponin was not significantly associated with ESUS after adjusting for confounders, suggesting that a cardio-embolic cause may not be the dominant mechanism in this group.

\section{BRAIN AGING AND CARDIOVASCULAR RISK FACTORS IN CHRONIC HIV: A LONGITUDINAL MRI STUDY}

\begin{abstract}
1,2,3 David Jakabek, ${ }^{2,3}$ Caroline D Rae, 1,2,3,4Bruce J Brew, 1,2,5 Lucette A Cysique. ${ }^{1}$ Departments of Neurology and HIV Medicine, St Vincent's Hospital, and Peter Duncan Neurosciences Unit, St Vincent's Centre for Applied Medical Research, Sydney, NSW, Australia; ${ }^{2}$ Neuroscience Research Australia, Sydney, NSW, Australia; ${ }^{3}$ Faculty of Medicine, University of New South Wales, Sydney, NSW, Australia; ${ }^{4}$ Faculty of Medicine, University of Notre Dame, Sydney, NSW, Australia; ${ }^{5}$ UNSW Psychology, University of New South Wales, Sydney, NSW, Australia
\end{abstract}

\subsection{6/bmino-2021-ANZAN.30}

Objectives We aimed to examine the relative contributions of HIV infection, age, and cardiovascular risk factors to subcortical brain atrophy.

Methods Virally suppressed HIV+ participants with low neuropsychological confounds $(\mathrm{n}=75)$ and demographically matched HIV- controls ( $\mathrm{n}=31$ ) completed baseline and 18month follow-up MRI scans, neuropsychological evaluation, cardiovascular assessments, and laboratory tests. HIV + participants were evaluated for HIV associated neurocognitive disorder (HAND). Subcortical volumes were extracted with Freesurfer. Volumetric and shape analyses were conducted using linear mixed-effect models incorporating interactions between age, time, and each of HIV status, HAND status, HIV disease factors, and cardiovascular markers.

Results HIV+ participants had smaller volumes of most structures compared to HIV- participants. Premature aging was evident in the pallidum using volumetric $(p=0.032)$ and shape analyses. Accelerated aging was observed in the caudate volumes for the more severe HAND subgroup $(\mathrm{p}=0.008)$ and was associated with longer HIV duration for putamen volumes $(\mathrm{p}=0.04)$. Higher CD4 counts had a protective effect on hippocampal volumes in older participants $(p=0.04)$. Cardiovascular measures were associated with smaller volumes across time for most structures; only the putamen demonstrated 